\title{
Prevalence and correlates of HIV infection among men who have sex with men: a multi-provincial cross-sectional study in the southwest of China
}

This article was published in the following Dove Press journal: HIVIAIDS - Research and Palliative Care

\author{
Mengliang $\mathrm{Ye}^{\prime}$ \\ Mohan Giri² \\ 'Department of Epidemiology and \\ Health Statistics, School of Public \\ Health and Management, Chongqing \\ Medical University, Chongqing 400016, \\ China; ${ }^{2}$ Department of Respiratory \\ Medicine, The First Affiliated Hospital \\ of Chongqing Medical University, \\ Chongqing 400016, China
}

Objective: This study aims to assess the prevalence and correlates of HIV Infection among men who have sex with men (MSM) in two major provinces in southwest of China.

Method: We adopted the method of anonymous survey and HIV blood test on 1,245 MSM $>18$ years of age from Chongqing and Sichuan Province, China.

Results: The prevalence of HIV infection among MSM was 21.20\%. Multiple logistic regression analysis revealed significant independent predictors of HIV infection among MSM as follows: lower level of education, preference to be receptive, lower frequency of condom use, drug usage, higher number of sexual partners in the past 1 month, and people suffering from sexually transmitted diseases. The distribution of HIV infection probability scores among HIV-negative MSM shows a positively skewed distribution, which will help to find persons at higher risk of HIV infection.

Conclusion: HIV prevalence in Chongqing and Sichuan Province is extremely high. We must find a way to identify people at high risk of HIV infection and employ different interventions among different risk levels of HIV infection in order to achieve maximum prevention and treatment benefit. Considering the relative shortage of resources, efforts to prevent HIV might be more effectively employed if they stratify men based on validated risk criteria.

Keywords: HIV/AIDS, men who have sex with men, risk factors, sexual behaviors, China

\section{Introduction}

Over the past 10 years, the prevalence of HIV among the general population has remained stable or even has fallen, however, there is significant increase in the rate of HIV transmission among men who have sex with men (MSM) in China. ${ }^{1}$ The prevalence of HIV among Chinese MSM has substantially increased from $1.4 \%$ in 2005 to $8 \%$ in $2015 .^{2}$ Furthermore, HIV cases caused by homosexual transmission have rapidly increased in the last decade, from $12.5 \%$ in 2007 to $25.8 \%$ in $2014 .{ }^{3}$ Although there are no precise statistics available on the number of infected MSM in China, it is clear that sexual transmission is the most prevalent method of transmission, with sexual transmission between men increasing markedly. ${ }^{4}$ According to the national sentinel surveillance data, the total HIV antibody positive rate of MSM was 7.3\% in 2013, total HIV antibody positive rate of injection drug users was $6.3 \%$, the rate of HIVinfected infants born to women living with HIV/AIDS was $6.7 \% .{ }^{1,4} \mathrm{HIV}$ is a disease of major public health concern in southwest of China, but there is a substantial lack of data to facilitate evidence-based decision making and planning to prevent new HIV infections among MSM.
School of Public Health and Management, Chongqing Medical University, Yixue Yuan Lu, Yuzhong District, Chongqing 400016, China

Tel $+86 \quad 15310939053$

Fax +862368485008

Email864313137@qq.com 
Therefore, a clear understanding of the risk factors for HIV infection among MSM, creation of a model of infection probability among MSM, and calculation of the probability of HIV infection among HIV-negative MSM is essential for effective implementation of HIV intervention measures among MSM. The establishment of a clinical prediction model is an efficient way to identify the higher-risk individuals to provide effective HIV treatment and improve HIV prevention. ${ }^{5}$ There are many studies regarding the prevalence of HIV and risk factors of HIV infection in MSM in southwest of China. Previous studies have combined HIV risk factors with a risk assessment and classification of HIV infection probability in MSM.6-8 Efforts to obtain HIV prevalence among MSM population in this region of China will potentially provide the information needed for policy decision making and refining prevention measures for high-risk people, for example, the pre-exposure prophylaxis (PrEP) offers a promising new approach to HIV prevention strategy., ${ }^{9,10}$ The present study aimed to assess the HIV prevalence rate and identify risk factors for HIV infection among MSM in two major provinces in southwest of China.

\section{Methods}

\section{Participants}

The current study is a baseline survey of an intervention study, designed to investigate patient compliance as well as the effect of PrEP, and to determine why there is poor compliance among MSM in Chongqing and Sichuan Province. The participants of this study were recruited using convenience sampling from March 2013 to September 2014 in Chongqing (Chongqing and Wanzhou) and Sichuan Province (Mianyang, Nanchong, Suining, and Yibin). Random sampling was not employed since MSM are hard to reach because of the stigmatization of homosexuality.

We adopted the method of anonymous survey. Investigators gave item-by-item explanations for each question as the participants completed the questionnaire. We also collected their blood sample for HIV testing at the same time. The specific implementation process included as follows: 1) survey preparation: recruited a research team, designed a research project, wrote a questionnaire, trained investigators, recruited MSM, and finally, held a kick-off meeting for staff of Centers for Disease Control (CDCs), non-governmental organization (NGO) members and other relevant members. 2) On-site survey: there were two survey sites, one was the NGO HOMO studio, the second being an affiliated hospital of Chongqing Medical University. Both places had individual interview rooms. The investigators introduced the purpose and significance of the survey to the participants, emphasizing the confidentiality of the survey. We adopted an anonymous survey method to ensure true answers to those sensitive issues. The investigators confirmed the answers and signed each questionnaire. The respondents received 50 Chinese yuan (CNY) for transportation and missed wages, as well as small gifts (towel and soap) for their participation.

\section{Ethical approval}

This study was reviewed and approved by the medical ethics committee of Chongqing Medical University and the review board of Chinese CDC (IRB Number: 2012010). Written informed consent was obtained from all participants prior to their enrollment in this study.

\section{Measures}

A structured interview assessment was designed to collect data and validated by previous studies. ${ }^{8,11}$ Variables and scales included demographic and background variables, sexual history and behaviors, HIV knowledge, HIV testing and counseling, perceived risk, and AIDS phobia. The outcome variable was HIV infection. To increase the validity of data and decrease recall bias, the activities of MSM over the past 3 to 6 months was used for assessment. ${ }^{12,13}$

\section{Demographic and background variables}

Sociodemographic variables included as follows: age, household registration (urban/rural), ethnicity (Han majority/ minorities), education (college or above, junior high or senior high, illiteracy/primary), marital status (single/never married), employment status (employed, retired, student), average monthly income, drinking habits in the past 6 months (yes/ no), and drug abuse (yes/no) in the past 6 months.

\section{Sexual history and behaviors}

An evaluation of HIV risk characteristics of the participants included number of male sex partners $(0 / \geq 1)$, preferred role in anal sex (insertive, almost equally, receptive), we compared those who reported either never using a condom or using condoms sometimes, with those who reported using condoms every time, finding sexual partners through the Internet (yes/ no), sexually transmitted disease (STD) (yes/no), and paying or being paid for sex (yes/no).

\section{HIV knowledge, HIV testing, and counseling}

HIV knowledge was assessed by a 13 -item scale, ${ }^{11}$ to determine the participants' knowledge about HIV and its transmission routes and prevention methods. Participants were 
presented with statement about HIV (for example, "a person using antibiotics cannot be infected by HIV.") and asked if they were true or false. Also, the participants could answer "don't know" if they were not sure. Items were scored dichotomously (one point for correct answers and zero for incorrect ones or “don't know”). Cronbach's alpha was 0.778 for the 13 items. Participants were also asked "Have you ever been tested for HIV?" and "Did you ever get HIV counseling in the past?" These two items could be answered as "yes" or "no".

\section{Perceived risk}

The participants' perception of risk was assessed by three items. The participants were asked: "How serious do you think HIV/AIDS is?", "How high do you think the prevalence rate of HIV is among MSM in your community?", and "How much do you think you are at risk of being infected with HIV by a sexual contact?". These items were rated on a 5-point Likert scale ("not at all" to "very much/high").

\section{HIV laboratory testing}

Blood sample was collected from each participant and tested for HIV. ELISA, (Wantai Biological Medicine Company, Beijing, China) as well as HIV1 +2 antibody diagnostic kit (colloid se method; origin, Japan) were used for HIV screening. ${ }^{14}$ If both results were negative, the participant was considered HIV-negative; if both results were positive, the participant was considered HIV-positive; if the results were inconsistent, the tests were repeated. In this study, the results were initially $99 \%$ consistently positive.

\section{Statistical analysis}

Data analysis was performed using SAS 9.2. Descriptive statistics, using mean, median, $\mathrm{SD}$, interquartile, frequency, and percentage were calculated. Comparative analyses, such as $\chi^{2}$ test was done. To identify the cofactors independently associated with the behaviors HIV infection; we used multiple logistic regression models in which we entered all variables that were associated with the outcome in the bivariate analysis at a $P$-value $\leq 0.05$. A total of $80 \%$ of the participants were in the modeling sample and $20 \%$ were in the model validity test and evaluation. Forecasting accuracy and the area under the receiver operating characteristic (ROC) curve were used to evaluate the accuracy of the prediction model. The probability of HIV infection among the HIV-negative participants was predicted by the established logistic regression model. The bound was set at 0.5 and if the predicted score was $>0.5$, then the predicted result was regarded as positive; otherwise, it was negative.
Furthermore, the predicted results were compared with antibody detection results.

\section{Results}

\section{Demographics and background variables}

Table 1 demonstrates sociodemographic characteristics of MSM in Chongqing and Sichuan Province. The mean age of the participants was 31.60 years $(\mathrm{SD}=9.02)$. A total of $28.59 \%$ of the participants were $\leq 25$ years, $51.97 \%$ between 26 and 40 years, and $19.44 \% \geq 40$ years of age. A total of $62.49 \%$ were urban residents, $97.35 \%$ of the Han race, and $50.60 \%$ of participants had a college education or above. A total of $69.16 \%$ were single, $80.32 \%$ were employed, and $61.45 \%$ had an income $\leq 3,000 \mathrm{CNY}$ per month. A total of $67.85 \%$ had a history of drinking, $1.07 \%$ had a history of taking illegal drugs in the past 6 months.

\section{Sexual history and behaviors}

In terms of sexual practice, in the past 1 month and 6 months, $12.85 \%$ and $49.35 \%$, respectively reported more than one sexual partner. In addition, $22.76 \%$ reported having had female sexual partners. Regarding sex role, $45.79 \%$ of participants were exclusively or predominantly engaged in insertive anal intercourse (IAI), 26.90\% exclusively or predominantly engaged in receptive anal intercourse, and $27.31 \%$ preferred both equally.

Consistent condom use in the past 6 months was reported by $52.45 \%$ of participants, while $47.55 \%$ reported never/ sometimes using condoms. A total of $40.27 \%$ reported finding sexual partners via the Internet and $5.57 \%$ had traded sex for money in the past 6 months. A total of $8.18 \%$ reported STDs (Table 1).

\section{HIV knowledge, HIV testing, and counseling}

The mean score of HIV knowledge was 5.88 ( $\mathrm{SD}=2.12$, range from 0 to $13,95 \% \mathrm{CI}$ : $5.76-6.00)$. A total of $64.42 \%$ of participants reported having ever had HIV testing. Only $46.91 \%$ reported ever having participated in HIV consulting (Table 1).

\section{Perceived risk}

A total of $91.81 \%$ of participants thought that AIDS was a serious or very serious disease. A total of $57.19 \%$ thought that the prevalence rate of HIV among MSM in their community was high or very high. A total of $81.84 \%$ thought that the risk of being infected with HIV via sexual contact was high or very high (Table 1$)$. 
Table I Demographics and risk factors among MSM in Chongqing and Sichuan Province, China

\begin{tabular}{|c|c|c|c|c|}
\hline Variables & All $(n, \%)$ & HIV+ (n, \%) & Prevalence (\%) & $P$-value ${ }^{a}$ \\
\hline \multicolumn{5}{|l|}{ Demographics and background } \\
\hline Age (years) & & & & 0.037 \\
\hline$\leq 25$ & $356(28.59)$ & $63(23.98)$ & 17.70 & \\
\hline $26-39$ & 647 (51.97) & $137(5 \mid .89)$ & 21.17 & \\
\hline$\geq 40$ & $242(19.44)$ & $64(24.24)$ & 26.44 & \\
\hline Household registration & & & & $<0.001$ \\
\hline Urban & 778 (62.49) & I $38(52.27)$ & 17.74 & \\
\hline Rural & $467(37.5 I)$ & $126(47.73)$ & 26.98 & \\
\hline Ethnicity & & & & 0.196 \\
\hline Han majority & $1,212(97.35)$ & $260(98.48)$ & 21.45 & \\
\hline Minorities & $33(2.65)$ & $4(1.52)$ & 12.12 & \\
\hline Education & & & & $<0.001$ \\
\hline College or above & $630(50.60)$ & $99(37.50)$ & $|5.7|$ & \\
\hline Junior high or senior high & $552(44.34)$ & $144(54.55)$ & 26.08 & \\
\hline Illiteracy/primary & $63(5.06)$ & $21(7.95)$ & 33.33 & \\
\hline Marital status & & & & $<0.001$ \\
\hline Single & $861(69.16)$ & $159(60.23)$ & 18.47 & \\
\hline Ever married & $384(30.84)$ & $105(39.77)$ & 27.34 & \\
\hline Occupation & & & & $<0.001$ \\
\hline Employed & $1,000(80.32)$ & $215(81.44)$ & 21.50 & \\
\hline Retired & $12(0.96)$ & $3(1.14)$ & 25.00 & \\
\hline Student & $139(11.16)$ & $14(5.3)$ & 10.07 & \\
\hline Unemployed & $94(7.55)$ & $32(12.12)$ & 34.04 & \\
\hline Income, CNY & & & & 0.003 \\
\hline$\leq 3,000$ & $765(61.45)$ & $183(69.32)$ & 23.92 & \\
\hline$>3,000$ & $480(38.55)$ & $81(30.68)$ & 16.88 & \\
\hline Drinking & & & 20.71 & 0.508 \\
\hline Yes & $840(67.85)$ & $174(66.16)$ & 22.36 & \\
\hline No & $398(32.15)$ & $89(33.84)$ & & \\
\hline Refused/missing & 7 & I & & \\
\hline Drug abuse in the past 6 months & & & 20.08 & 0.026 \\
\hline No & I, $199(98.93)$ & $249(94.32)$ & 46.15 & \\
\hline Yes & $13(1.07)$ & $6(2.35)$ & & \\
\hline Refused/missing & 33 & 9 & & \\
\hline \multicolumn{5}{|l|}{ Sexual history } \\
\hline \multicolumn{5}{|c|}{ Number of male sex partners in the past I month } \\
\hline$\leq 1$ & $977(87.15)$ & $190(73.08)$ & 19.45 & 0.002 \\
\hline$>1$ & $244(12.85)$ & $70(16.92)$ & 28.69 & \\
\hline Refused/missing & 24 & 4 & & \\
\hline \multicolumn{5}{|c|}{ Number of male sex partners in the past 6 months } \\
\hline$\leq 1$ & $587(50.65)$ & $|2|(48.02)$ & 20.06 & 0.345 \\
\hline$>1$ & $572(49.35)$ & $13 \mid(51.98)$ & 22.90 & \\
\hline Refused/missing & 86 & 12 & & \\
\hline \multicolumn{5}{|c|}{ Number of female sex partners in the past 6 months } \\
\hline 0 & $869(77.24)$ & $172(70.20)$ & 19.80 & 0.003 \\
\hline$\geq 1$ & $256(22.76)$ & $73(29.80)$ & 28.51 & \\
\hline Refused/missing & 120 & 19 & & \\
\hline \multicolumn{5}{|l|}{ Preferred position in anal sex } \\
\hline Insertive & $565(45.79)$ & $86(32.82)$ & 15.22 & $<0.001$ \\
\hline Almost equally & $337(27.31)$ & 89 (33.97) & 26.41 & \\
\hline Receptive & $332(26.90)$ & $87(33.21)$ & 26.20 & \\
\hline Refused/missing & 11 & 2 & & \\
\hline \multicolumn{5}{|l|}{ Condoms use in the past 6 months } \\
\hline Consistent & $557(52.45)$ & $108(46.35)$ & 19.40 & 0.028 \\
\hline Never/sometimes & $505(47.55)$ & $125(53.65)$ & 24.75 & \\
\hline Refused/missing & 183 & 31 & & \\
\hline
\end{tabular}

(Continued) 
Table I (Continued)

\begin{tabular}{|c|c|c|c|c|}
\hline Variables & All $(n, \%)$ & HIV+ (n, \%) & Prevalence (\%) & $P$-value ${ }^{a}$ \\
\hline \multicolumn{5}{|c|}{ Finding sex partners on internet } \\
\hline No & $700(59.73)$ & $142(55.69)$ & 20.29 & 0.137 \\
\hline Yes & $472(40.27)$ & II $3(44.3 \mathrm{I})$ & 23.94 & \\
\hline Refused/missing & 73 & 9 & & \\
\hline \multicolumn{5}{|c|}{ Sexually transmitted diseases } \\
\hline No & I, I 34 (9I.82) & $23 \mid(88.17)$ & 20.37 & 0.015 \\
\hline Yes & $101(8.18)$ & $31(11.83)$ & 30.69 & \\
\hline Refused/missing & 10 & 2 & & \\
\hline \multicolumn{5}{|c|}{ Trading sex in the past 6 months } \\
\hline No & $1,169(94.43)$ & $246(93.18)$ & 21.04 & 0.320 \\
\hline Yes & $69(5.57)$ & $18(6.82)$ & 26.09 & \\
\hline Refused/missing & 7 & 0 & & \\
\hline \multicolumn{5}{|c|}{ HIV testing and counseling } \\
\hline \multicolumn{5}{|l|}{ HIV testing } \\
\hline No & $443(35.58)$ & II $3(42.80)$ & 25.51 & 0.006 \\
\hline Yes & $802(64.42)$ & $15 \mathrm{I}(57.20)$ & 18.83 & \\
\hline \multicolumn{5}{|l|}{ HIV counseling } \\
\hline No & 661 (53.09) & $143(54.17)$ & 21.63 & 0.693 \\
\hline Yes & $584(46.91)$ & $|2|(45.83)$ & & \\
\hline \multicolumn{5}{|c|}{ Perceived risk and AIDS phobia } \\
\hline \multicolumn{5}{|c|}{ How serious do you think HIVIAIDS is? } \\
\hline Very serious & $833(66.91)$ & $17 \mid(64.77)$ & 20.53 & 0.242 \\
\hline Serious & $310(24.90)$ & $63(23.86)$ & 20.32 & \\
\hline Moderate & $78(6.27)$ & $21(7.95)$ & 26.92 & \\
\hline Not serious & $14(1.12)$ & $7(2.65)$ & 50.00 & \\
\hline Not at all & $10(0.80)$ & $2(0.76)$ & 20.00 & \\
\hline \multicolumn{5}{|c|}{ Prevalence rate of HIV among MSM in your community? } \\
\hline Very high & $245(19.68)$ & $49(18.56)$ & 20.00 & 0.415 \\
\hline High & $467(37.51)$ & $95(35.98)$ & 20.34 & \\
\hline Moderate & $34 I(27.39)$ & $79(29.92)$ & 23.17 & \\
\hline Low & $113(9.08)$ & $23(8.7 \mathrm{I})$ & 20.35 & \\
\hline Very low & $79(6.35)$ & $18(6.82)$ & 22.78 & \\
\hline \multicolumn{5}{|c|}{ Risk of being infected by HIV via sexual contact? } \\
\hline Very high & $762(61.2)$ & $162(61.36)$ & 21.26 & 0.915 \\
\hline High & $257(20.64)$ & $56(21.21)$ & 21.79 & \\
\hline Moderate & $141(11.33)$ & $27(10.23)$ & 19.15 & \\
\hline Low & $45(3.61)$ & $10(3.79)$ & 22.22 & \\
\hline Very low & $40(3.21)$ & $9(3.4 I)$ & 22.50 & \\
\hline
\end{tabular}

Notes: ${ }^{a} \chi 2$ test was applied if variables were categorical.

Abbreviation: MSM, men who have sex with men.

\section{Factors associated with HIV seropositivity among MSM}

A total of 1,245 MSM were tested for HIV, of which 264 were HIV-positive. The total antibody positive rate was $21.20 \%$ (264/1,245, 95\% CI: $18.95 \%-23.47 \%)$, which was significantly higher than the national average rate of $8.0 \%$ $(P<0.05) .{ }^{3}$ Logistic regression analysis showed that the main factors associated with HIV infection were level of education (illiteracy/primary vs college or above, adjusted OR [AOR] 2.57; 95\% CI: 1.28-5.13), preferred role in anal sex ("receptive" vs "insertive", AOR 2.58; 95\% CI: 1.75-3.80), condom use in anal intercourse (never/sometimes vs always,
AOR 1.68; 95\% CI: 1.11-2.56), drug abuse (AOR 3.40; 95\% CI: 1.28-8.99), number of male sexual partners (AOR 1.59; 95\% CI: 1.08-2.33), and suffering from STDs (AOR 1.76; 95\% CI: 1.05-2.95) (Table 2).

\section{Accuracy evaluation and validation results}

The validation sample included 248 participants, including 52 who were HIV positive (20.97\%) and 196 HIV negative (79.03\%). We used logistic regression model to calculate estimated HIV infection probability of each MSM. When the value of probability was $>0.5$, then the prediction was considered as HIV positivity; otherwise, the prediction was 
Table 2 Risk factors associated with HIV infection among MSM in Chongqing and Sichuan Province

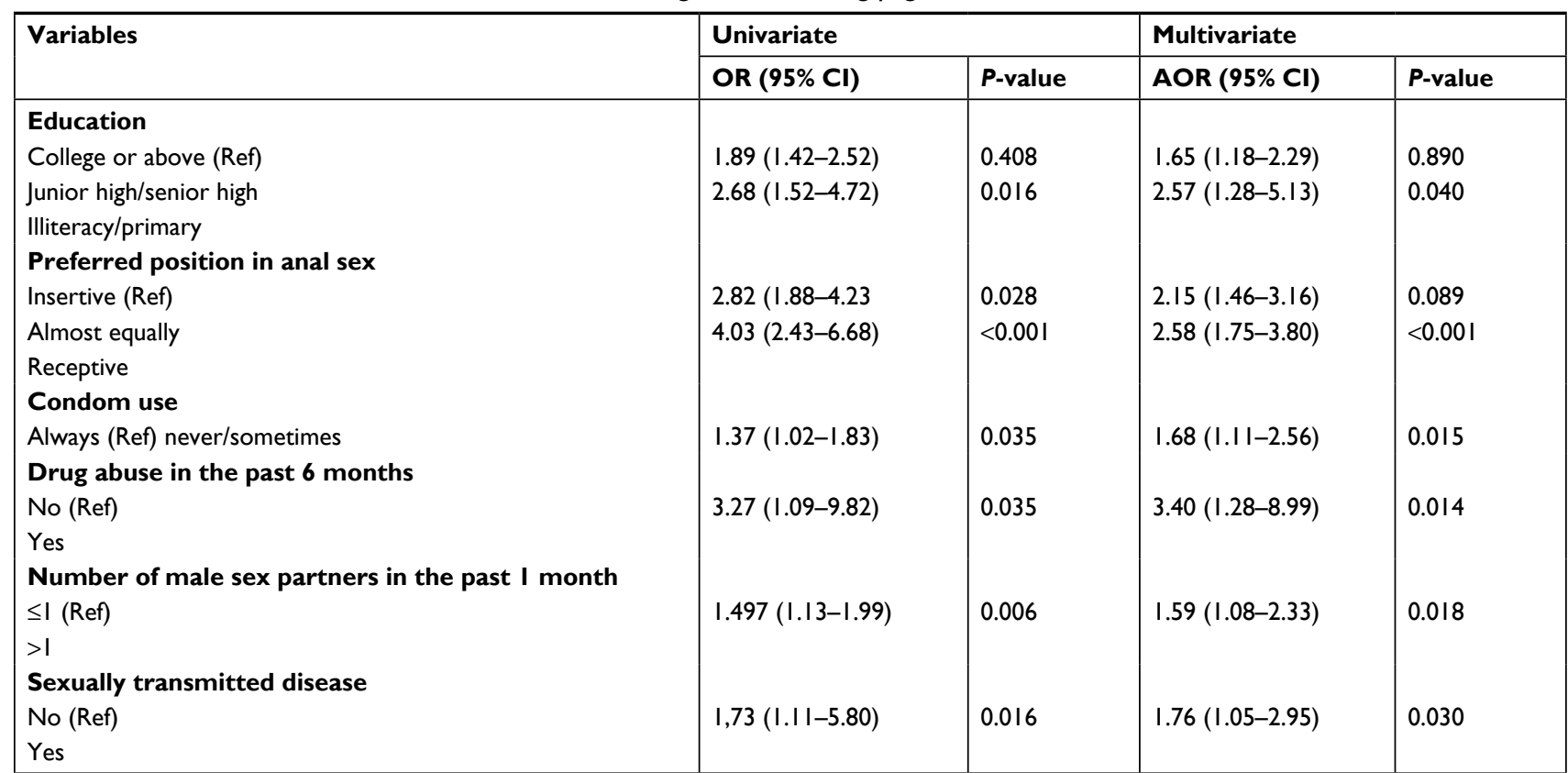

Abbreviations: MSM, men who have sex with men; OR, odds ratio; AOR, adjusted OR; Ref, reference.

regarded as HIV negativity. The predictions were then compared with the HIV antibody test. The results have shown a sensitivity of $54 \%$, specificity of $69 \%$, and rate of correct diagnosis of $66 \%$, and the area under the ROC curve measures $0.637, P<0.05$ (Figure 1).

\section{Probability prediction of HIV-negative MSM}

Nineteen HIV-negative MSM were excluded from the final analysis because of missing data, leaving 962 for the final prediction. The distribution of HIV infection probability among these 962 MSM is presented in Table 3 and Figure 2. The minimum value of HIV infection probability is 0.086 , the maximum value is 0.682 , and the average value is 0.201 . The median value is $0.195, \mathrm{P} 25$ is 0.134 , and $\mathrm{P} 75$ is 0.249 .

\section{Discussion}

The overall prevalence of HIV infection among MSM in Chongqing and Sichuan Province, China was found to be $21.20 \%$ (95\% CI: $18.95-23.47$ ), which is similar to the finding of previous studies. ${ }^{15,16}$ This finding was much higher than the national average of $8 \%$, and also higher than in other vulnerable groups, such as IV drug users (3.6\%). ${ }^{3}$ In the last decade, HIV prevalence progressively increased among MSM both in Chongqing (from 2006 to 2009, 10.9\%, $12.8 \%, 10.6 \%, 19.1 \%$, respectively) and in Chengdu, Sichuan Province (from $0.6 \%$ in 2003 to $16.3 \%$ in 2011)..$^{17,18}$ This is

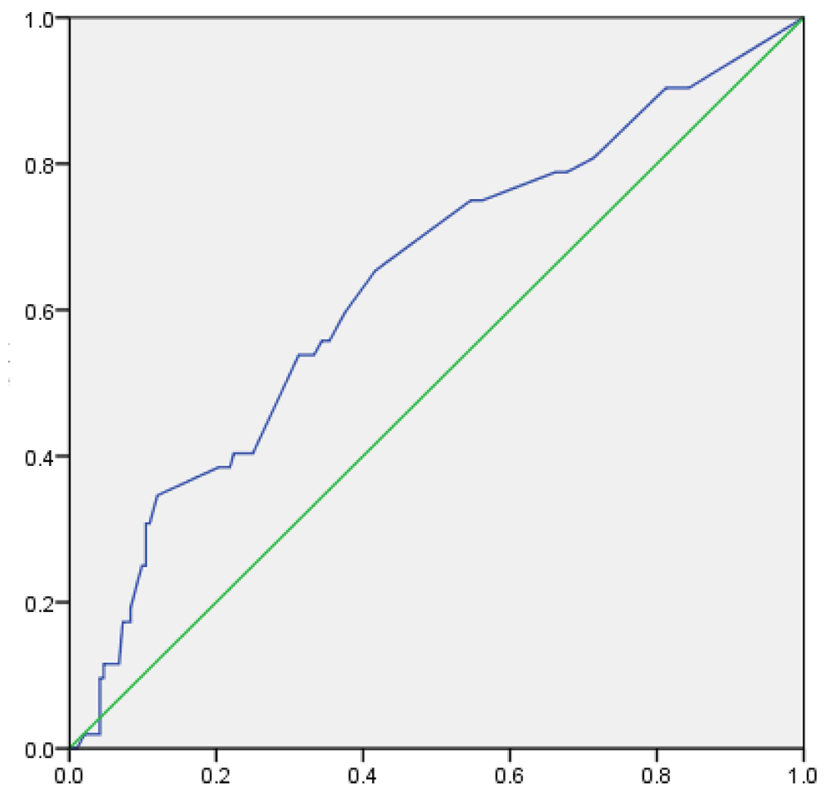

Figure I Receiver operating characteristic curve about the prediction effect of the logistic regression model.

consistent with the national trend of homosexual transmission of HIV. Sexual transmission is now the primary mode of transmission, with sexual transmission between men increasing markedly. ${ }^{3}$ The main reason for this increase is unprotected sex among MSM, leading to more diagnosed cases of HIV/ AIDS and, with earlier detection, longer life spans of HIVpositive MSM. ${ }^{1}$ Our findings underscore the imperative need 
Table 3 Distribution of estimated HIV infection probability in 962 MSM

\begin{tabular}{|l|l|l|l|l|l|l|l|}
\hline $\mathbf{n}$ & Minimum & Maximum & Average & Median & P25 & P75 & IQR \\
\hline 962 & 0.086 & 0.682 & 0.201 & 0.195 & 0.134 & 0.249 & 0.115 \\
\hline
\end{tabular}

Abbreviation: MSM, men who have sex with men

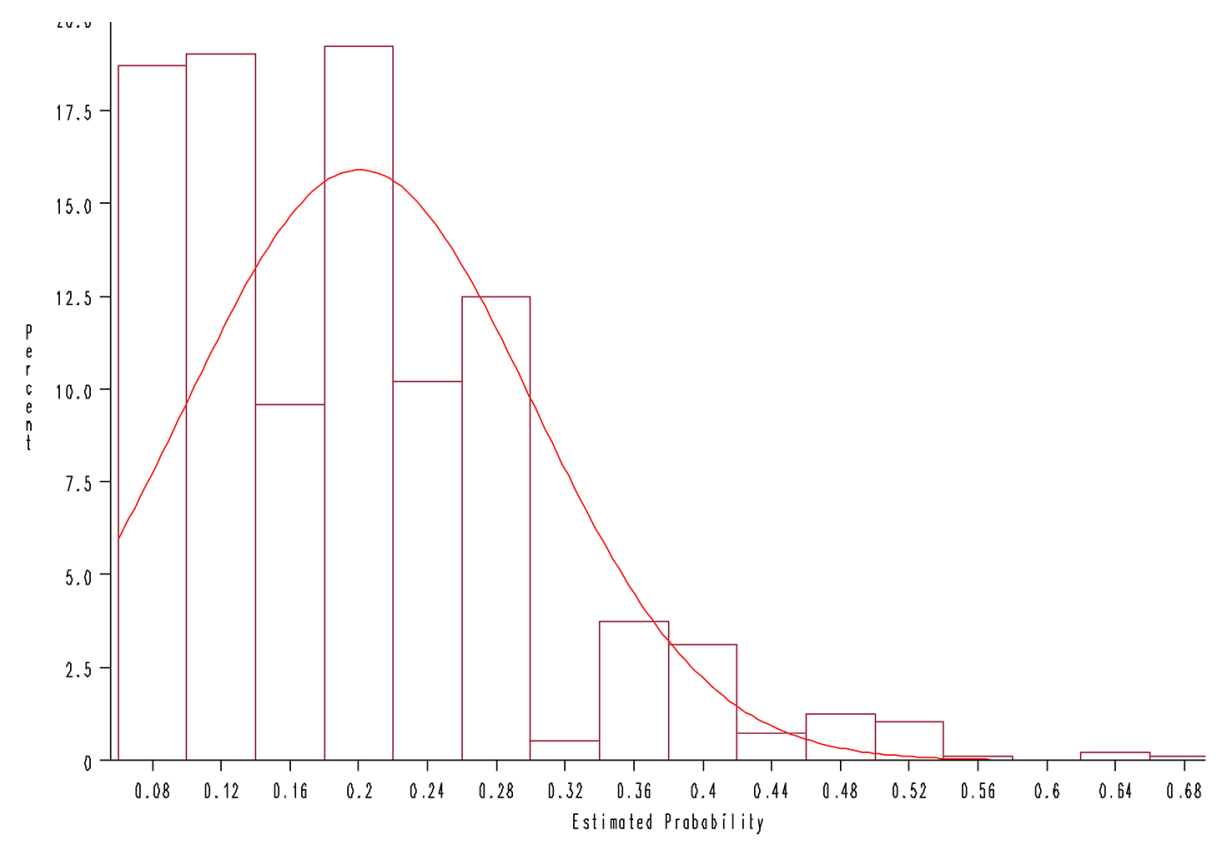

Figure 2 Distribution of HIV estimated probability among 962 MSM.

Abbreviation: MSM, men who have sex with men.

to strategically target HIV prevention intervention measures and treatment efforts for MSMs.

Single factor analysis indicated that older MSM were more likely to be diagnosed with HIV, as is to be expected: older MSM are more likely to have had more lifetime sexual contacts, and are therefore more likely to have been exposed to HIV. ${ }^{19}$ We also found that MSM with rural household registration, lower educational level, never-married MSM, lower average monthly income, and drug abuse were more likely to be HIV-positive. All of those factors can help us detect individuals who are vulnerable to HIV exposure. Sex role preference is also associated with HIV infection. ${ }^{20}$ Our findings also showed that MSM with receptive sexual roles are more likely to be HIV-positive. Multiple sex partners, infrequent condom use, and STDs have been associated with biological risk for HIV infection in MSM. ${ }^{21,22}$ Those who had never undergone HIV testing before were more likely to be diagnosed with HIV. Many researchers found that those who have sex with females might play a significant role in HIV transmission for MSM. ${ }^{23}$ In line with the findings of Beyrer et al, ${ }^{24}$ our finding suggests that MSM who also have sex with women have higher rates of HIV positivity than those who have sex only with men. Among our participants, $30.84 \%$ had a history of marriage and $22.76 \%$ had had sex with females; this is the bridge of HIV transmission between MSM and the general female population. The HIV prevalence in MSM who had female sex partners was $26.90 \%$, which is much higher than those who have had sex only with men.

Multiple logistic regression showed that low education level, preferred receptive role in anal sex, no use of condoms in anal sex, multiple sexual partners, and STDs were all independent risk factors for HIV infection. Men without a college education were $49.39 \%$ more likely to be diagnosed with HIV, perhaps due to their low awareness of HIV risk and high-risk sexual behaviors. Those preferring receptive anal sex were also more likely to be diagnosed with HIV; this may largely be because unprotected anal sex carries a higher risk of HIV transmission, ${ }^{17}$ while MSM preferring receptive anal sex wrongly perceived themselves to have a lower risk of HIV infection than those preferring insertive anal sex. ${ }^{25} \mathrm{MSM}$ who never or only sometimes used condoms during anal sex with their male sexual partner were more likely to be HIV- 
positive. In our study, only $52.45 \%$ of MSM used condoms on a regular basis, while the national average $66.5 \%$ in 2007$)^{26}$ and other countries had a much higher percentage of MSM using condoms on a regular basis (75\% among HIV-positive MSM in concordant and $81 \%$ in discordant; $73 \%$ among HIV-negative MSM in concordant and $87 \%$ in discordant in 2014). ${ }^{27}$ This lack of condom use by our study participants was, of course, associated with a much higher rate of HIV positivity. Those who had drug abuse in the past 6 months also had a much higher prevalence of HIV infection than those who did not. Our study also showed that having multiple male sex partners was associated with HIV infection. The participants in this study had an average of 2.02 male partners in the past 6 months. Of great concern, more than $10 \%$ reported having at least five male partners during the same period. STDs have been associated with biological risk for HIV infection in MSM. ${ }^{21,22}$ A total of $8.18 \%$ of our participants reported that they had been diagnosed with STDs. The finding of common place unprotected anal intercourse, multiple sex partners, drug abuse, and STDs suggests an extremely high risk of continuous rapid expansion of the HIV epidemic among MSM in Chongqing and Sichuan Province.

The mean of anticipated HIV infection probability among HIV-negative MSM was 20.1\%, and the distribution of HIV infection probability was positively skewed and uneven. This indicates that we should find ways to identify MSM with high levels of risk factors and use different intervention measures aimed at groups with different risk levels in order to get the best prevention and treatment benefits (for example, identifying appropriate PrEP candidates among MSM) ${ }^{28}$ The fitness of this model in HIV infection is good, no co-linearity problem is shown, and the effect of prediction is good, with the area under the ROC curve being 0.637.

Our research had several limitations. First, we used the baseline data of a prevention trial. Those people who volunteered to join the prevention trial may have had high risk perception, may have had extremely high-risk sexual behaviors, which are, of course, associated with a high prevalence of HIV. Second, sexual behaviors were self-reported and thus may have been underestimated due to the sensitive nature of the questions. Third, the character of the crosssectional design precluded the ascertainment of causality; more research on HIV prevalence and risk factors predicting new infections among MSM in China is needed. ${ }^{29}$ Fourth, convenience sampling provides insufficient power to detect differences among sociodemographic subgroups and produces estimates that lack generalizability to any identifiable target population or subpopulations (except for the sample studied). Thus, our results may not be generalized since we employed cross-sectional design and a small sample at one site. Further studies should utilize the random driven sampling (RDS) at multiple sites to collect more participants. We also found that MSM being minority people, drug users and unemployed, low-income and illiterate people to access HIV diagnosis were more likely to be HIV-positive. This may be due to MSM are among more social vulnerable groups and, they have disadvantageous access for health care and HIV diagnosis. Finally, participants were recruited only from Chongqing and Sichuan Province; we should collect data on MSM in other provinces. We should keep on refining the model to be able to counsel MSM on the most effective HIV prevention measures.

\section{Conclusion}

In summary, the HIV epidemic situation is very serious among MSM in Chongqing and Sichuan areas and the AIDS control workload is heavy. The common practice of risky sexual behaviors among MSM (multiple anal sexual partners, nonuse of condoms, and preferred receptive role in anal sex) highlight the urgent need for an effective and comprehensive HIV prevention strategy that includes traditional HIV behavioral interventions, such as HIV/AIDS education and promotion of voluntary counseling and testing services, safer sexual behaviors, consistent condom use, and treatment of STDs, as well as alternative biomedical interventions, such as PrEP. ${ }^{30}$

\section{Acknowledgments}

This study was supported by Chongqing Science and Technology Commission of the People's Republic of China (Grant no.cstc2017jcyjAX0185). The authors express their thanks to individuals who volunteered to participate in this study and Chinese Center for Disease Control and Prevention, Chongqing, China for their contributions to the project.

\section{Disclosure}

The authors report no conflicts of interest in this work.

\section{References}

1. Ministry of Health of the People's Republic of China. 2012 China AIDS Response Progress Report. Unaids [Internet]; 2012; 1-70. Available from: http://www.unaids.org/en/dataanalysis/knowyourresponse/cou ntryprogressreports/2012countries/ce_CN_Narrative_Report[1].pdf. Accessed June 11, 2018.

2. Wu Z. [Achievement of HIV/AIDS program in the past 30 years and challenges in China]. Vol. 36, Zhonghua liu xing bing xue za zhi $=$ Zhonghua liuxingbingxue zazhi. China. 2015:1329-1331. Chinese.

3. UNAIDS. 2015 China AIDS response progress report. 2015;(May):126. Available from: http://www.unaids.org/sites/default/files/country/ documents/CHN_narrative_report_2015.pdf. Accessed June 11, 2018. 
4. Zhou Y, Li D, Lu D, Ruan Y, Qi X, Gao G. Prevalence of HIV and syphilis infection among men who have sex with men in China: a meta-analysis. Biomed Res Int. 2014;2014:620431-12.

5. Menza TW, Hughes JP, Celum CL, Golden MR. Prediction of HIV acquisition among men who have sex with men. Sex Transm Dis. 2009;36(9):547-555.

6. Sandfort TG, Lane T, Dolezal C, Reddy V. Gender Expression and Risk of HIV Infection Among Black South African Men Who Have Sex with Men. AIDS Behav. 2015;19(12):2270-2279.

7. Ruan Y, Li D, Li X, et al. Relationship between syphilis and HIV infections among men who have sex with men in Beijing, China. Sex Transm Dis. 2007;34(8):592-597.

8. Feng Y, Wu Z, Detels R, et al. HIV/STD prevalence among men who have sex with men in Chengdu, China and associated risk factors for HIV infection. J Acquir Immune Defic Syndr. 2010;53(Suppl 1):S74-S80.

9. Okwundu CI, Uthman OA, Okoromah CA. Antiretroviral pre-exposure prophylaxis (PrEP) for preventing HIV in high-risk individuals. Cochrane Database Syst Rev. 2012;7(7):CD007189.

10. Mcgowan I. An overview of antiretroviral pre-exposure prophylaxis of HIV infection. Am J Reprod Immunol. 2014;71(6):624-630.

11. Zhou F, Gao L, Li S, et al. Willingness to accept HIV pre-exposure prophylaxis among Chinese men who have sex with men. PLoS One. 2012;7(3):e32329.

12. Jemmott LS, Jemmott JB, O'Leary A. Effects on sexual risk behavior and STD rate of brief HIV/STD prevention interventions for African American women in primary care settings. Am J Public Health. 2007;97(6):1034-1040.

13. Kalichman SC, Simbayi L. Traditional beliefs about the cause of AIDS and AIDS-related stigma in South Africa. AIDS Care. 2004; 16(5):572-580

14. van den Berk GE, Frissen PH, Regez RM, Rietra PJ. Evaluation of the rapid immunoassay determine HIV $1 / 2$ for detection of antibodies to human immunodeficiency virus types 1 and 2. J Clin Microbiol. 2003;41(8):3868-3869.

15. Qi J, Zhang D, Fu X, et al. High risks of HIV transmission for men who have sex with men--a comparison of risk factors of HIV infection among MSM associated with recruitment channels in 15 cities of China. PLoS One. 2015;10(4):e0121267.

16. Wu Z, Xu J, Liu E, et al. National MSM Survey Group, et al. HIV and syphilis prevalence among men who have sex with men: a cross-sectional survey of 61 cities in China. Clin Infect Dis. 2013;57(2):298-309.

17. Zhang Y, Chen P, Lu R, et al. Prevalence of HIV among men who have sex with men in Chongqing, China, 2006-2009: cross-sectional biological and behavioural surveys. Sex Transm Infect. 2012;88(6):444-450.
18. Wang L, Wang L, Norris JL, et al. HIV prevalence and influencing factors analysis of sentinel surveillance among men who have sex with men in China, 2003-2011. Chin Med J. 2012;125(11):1857-1861.

19. den Daas C, Goenee M, Bakker BH, de Graaf H, Op de Coul EL. Comparing databases: determinants of sexually transmitted infections, HIV diagnoses, and lack of HIV testing among men who have sex with men. BMC Public Health. 2015;15:1114.

20. Lou J, Wu J, Chen L, Ruan Y, Shao Y. A sex-role-preference model for HIV transmission among men who have sex with men in China. BMC Public Health. 2009;9(Suppl 1):S10.

21. Chin-Hong PV, Husnik M, Cranston RD, et al. Anal human papillomavirus infection is associated with HIV acquisition in men who have sex with men. AIDS. 2009;23(9):1135-1142.

22. Millett GA, Peterson JL, Wolitski RJ, Stall R. Greater risk for HIV infection of black men who have sex with men: a critical literature review. Am J Public Health. 2006;96(6):1007-1019.

23. Lau JT, Lin C, Hao C, Wu X, Gu J. Public health challenges of the emerging HIV epidemic among men who have sex with men in China. Public Health. 2011;125(5):260-265.

24. Beyrer C, Trapence G, Motimedi F, et al. Bisexual concurrency, bisexual partnerships, and HIV among Southern African men who have sex with men. Sex Transm Infect. 2010;86(4):323-327.

25. Jin F, Crawford J, Prestage GP. HIV risk reduction behaviours in gay men: Unprotected anal intercourse, risk reduction behaviours, and subsequent HIV infection in a cohort of homosexual men. AIDS 2009;23(2):243-252.

26. Yan X, Li Cm FL. Factors and changes of condom use among men who have sex with men in 16 Cities of China. Chinese JAids Std. 2008;14:2.

27. Paz-Bailey G, Mendoza MC, Finlayson T, et al; NHBS Study Group. Trends in condom use among MSM in the United States: the role of antiretroviral therapy and seroadaptive strategies. AIDS. 2016;30(12):1985-1990.

28. Wilton J, Kain T, Fowler S, et al. Use of an HIV-risk screening tool to identify optimal candidates for PrEP scale-up among men who have sex with men in Toronto, Canada: disconnect between objective and subjective HIV risk. J Int AIDS Soc. 2016;19(1):20777.

29. Dong Z, Xu J, Zhang H, et al; China National HIV Prevention Study Group. HIV incidence and risk factors in Chinese young men who have sex with men--a prospective cohort study. PLoS One. 2014;9(5) e97527.

30. Xiao Z, Li X, Mehrotra P. HIV/sexual risk reduction interventions in China: a meta-analysis. AIDS Patient Care STDS. 2012;26(10):597-613.
HIV/AIDS - Research and Palliative Care

\section{Publish your work in this journal}

HIV/AIDS - Research and Palliative Care is an international, peerreviewed open access journal focusing on advances in research in HIV its clinical progression and management options including antiviral treatment, palliative care and public healthcare policies to control viral spread. The journal is included in PubMed. The manuscript man-
Dovepress

agement system is completely online and includes a very quick and fair peer-review system, which is all easy to use. Visit http://www.dovepress. com/testimonials.php to read real quotes from published authors. 